\title{
Area Dependent Low Frequency Noise in Metal Oxide Based Resistive Random Access Memory
}

\author{
Zheng Fang, Xiang Li, Xinpeng Wang, and Patrick Guoqiang Lo
}

\begin{abstract}
Metal oxide based resistance random access memory (RRAM) has been extensively studied as one of the most promising candidate for next generation nonvolatile memory; however the current conduction mechanism is not yet clearly understood. To tackle this problem, low frequency noise behavior in metal oxide based RRAM device has been investigated in this work. Together with DC current voltage characteristics, it confirms that for the low resistance state, current conduction is localized without an area dependence, whereas, for the high resistance state, it is a uniform leakage current throughout the whole device area. This is consistent with the filament type resistive switching phenomenon in such devices.
\end{abstract}

Index Terms-Current conduction, low frequency noise, resistive switching memory

\section{INTRODUCTION}

Transition metal oxide materials have been extensively studied in the recent years for future generation nonvolatile memory application owing to promising performance and CMOS compatibility [1], [2]. Switching mechanism in this type of Resistive Random Access Memory devices is proposed to be related to a local oxygen vacancy filament formation and rupture by redox reaction [3], [4], which has been discussed in details in the previous chapter. However, the current conduction mechanisms of different resistance states are still under debate despite of its importance to understand the RRAM switching properties and power consumption [5].

The low frequency noise (LFN) measurement is a powerful tool to characterize the current conduction mechanism and fluctuation sources in any electronic devices [6]. Since the LFN increases reciprocally with device area, it has received wide attentions especially in aggressively scaled devices [6, 7]. For RRAM devices to be used as ultra-high density nonvolatile memory, LFN characteristics are expected to provide useful information about conduction and fluctuation mechanisms as well as defect characteristics [8]-[11]. In this chapter, current conduction mechanism is investigated with DC and LFN analysis on $\mathrm{HfO}_{x}$ based RRAM devices of various dimensions.

\section{DEVICE UNDER TEST}

$H f O_{x}$ based RRAM devices used for this study are

Manuscript received July 6, 2012; revised September 5, 2012.

The authors are with Institute of Microelectronics, A*STAR (Agency for Science, Technology and Research), Singapore CO 117685 (e-mail: fangz@ime.a-star.edu.sg) fabricated using the standard CMOS platform technologies on 8 inch Si wafers. $20 \mathrm{~nm}$ thick Ti and $50 \mathrm{~nm}$ thick Pt are firstly deposited by the E-beam evaporation as the adhesion layer and the bottom electrode, respectively. Then $10 \mathrm{~nm}$ $\mathrm{HFO}_{x}$ and $50 \mathrm{~nm} \mathrm{TiN}$ were deposited by reactive sputtering with proper annealing conditions as the respective switching layer and top electrode. Devices were then patterned with lithography and dry etched into the square shape with dimensions from $10 \times 10 \mu \mathrm{m}^{2}$ to $100 \times 100 \mu \mathrm{m}^{2}$. Post metal dielectrics were used to cover the wafer preventing devices from further possible damages after fabrication.

In this work, a group of measurements have been taken out on four types of square devices with side length of 10um, 20um, 50um and 100um (for clarity, denoted as L10, L20, L50 and L100 respectively). To reduce the possibility of measurement errors, for each area, at least 3 devices are used, and for each device, three states of fresh, HRS and LRS are measured with five different biases.

\section{RESUlTS AND DISCUSSION}

Device used for conduction study exhibits polarity dependent bipolar resistive switching behavior and a typical transition curve is show in Fig. 1. The resistance of the device can be changed between high resistance state (HRS) and low resistance state (LRS) by electrical voltage bias or current bias.

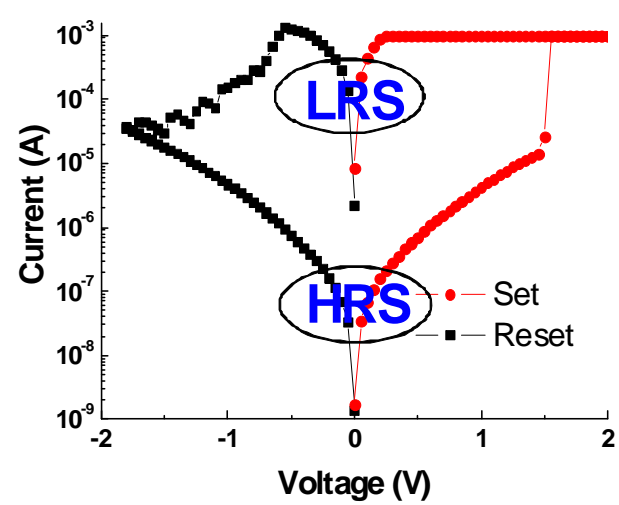

Fig. 1. Typical bipolar resistive switching curve for $H f O_{x}$ RRAM device used for low frequency noise analysis and current conduction investigation

To compare DC current levels in the devices with different areas, small bias voltage sweep is applied in the states of fresh, HRS and LRS respectively. Voltage below transition threshold is used to avoid resistive switching during measurement. To get a clearer picture of the resistance levels at different states with device area scaling, Fig. 2 is plotted. It is seen that in the graph, HRS resistance is very similar to that of fresh device, which indicates a roughly complete recovery of conduction filament during reset process. More than that, 
it shows a linear scaling resistance with device area in fresh and HRS states with log-log scale, while resistance keeps nearly constant at all device area points, which is believed to be related to compliance current level during set operations.

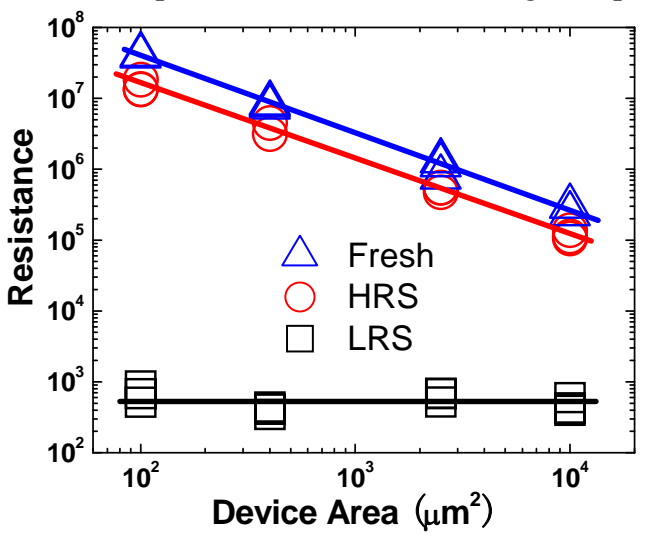

Fig. 2. Resistance versus device area plot for fresh, HRS and LRS. For both fresh and HRS state, resistance shows a reciprocal scaling effect with respect of device areas; while for LRS, there is no dependence on device areas.

Noise measurement are carried out on three states of device, fresh, HRS and LRS, and normalized power spectral density (PSD) with current is plotted in Fig. 3 under different bias conditions. It is seen that for all three states, PSD slopes are close to 1 , which confirms the $1 /$ f noise nature for the oxide-based RRAM devices. It can also be seen from the figure that the noise PSD is independent of bias conditions, which means it is an intrinsic characteristic of the current conduction.

Fig. 4 is the PSD spectrum of HRS state from L10 to L100. It is seen that the noise PSD scales reciprocally with the area (similar to the observation in CMOS devices). To compare L10 with L100, a PSD level difference of around two orders of magnitude is observed. However, after normalizing with the device area, the four PSD curves fall into a similar level, as illustrated in Fig.

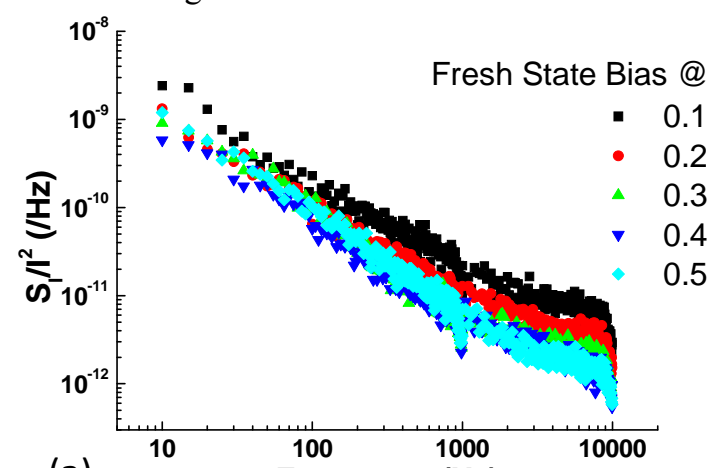

(a)

Frequency $(\mathrm{Hz})$

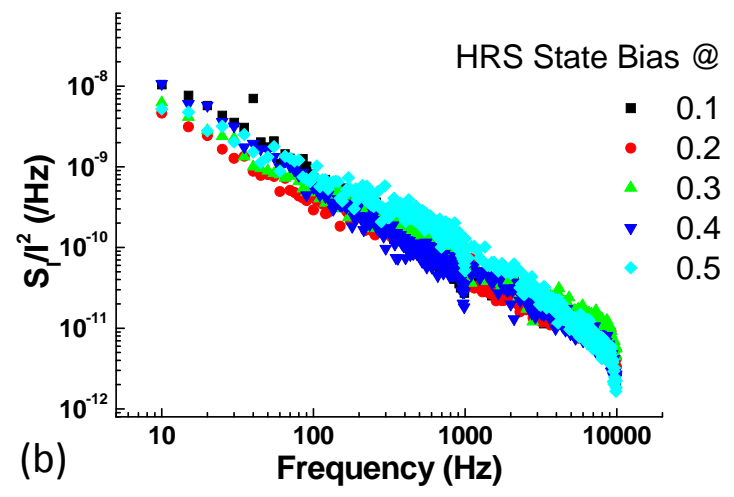

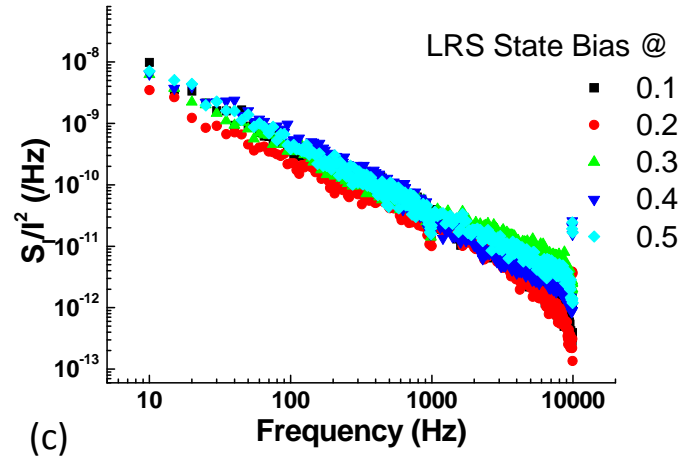

Fig. 3. Normalized PSD plot for (a) Fresh (b) HRS (c) LRS states under small bias. The noise PSD is independent of bias conditions for all three states.
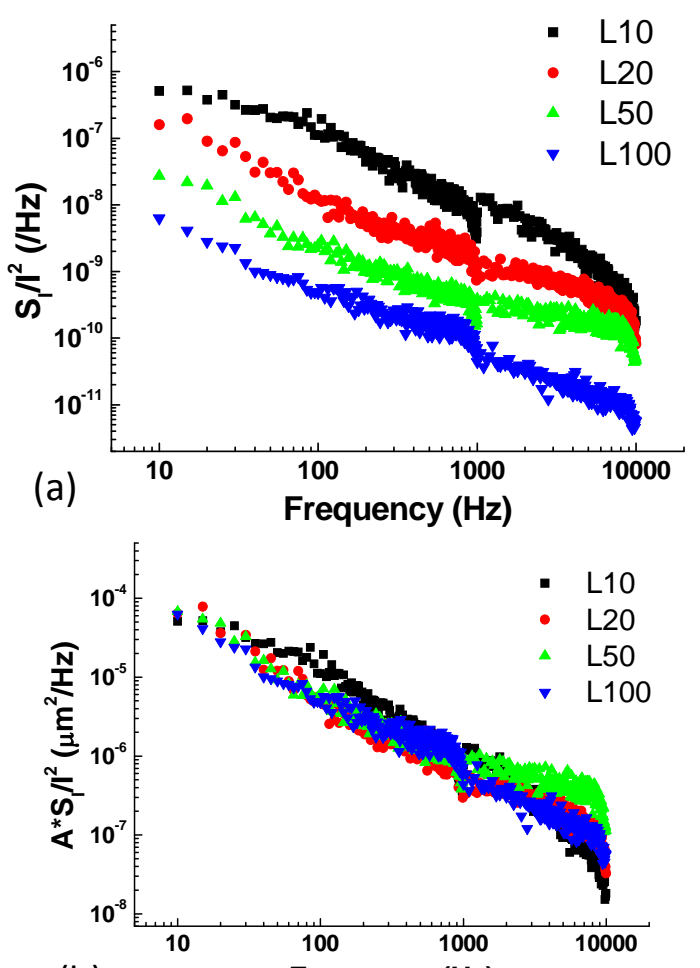

(b)

Frequency $(\mathrm{Hz})$

Fig. 4. (a) Measured noise PSD of HRS state for device L10 to L100. (b) Area normalization on HRS PSD spectrum results in a similar noise level for all devices.

Unlike fresh or HRS, LRS current conduction is localized with conduction filament; as so, LFN spectrum is expected to be independent of device areas. LFN study on different area devices are compared in Fig. 5. It is can be seen that LFN PSD is similar for all four devices with various areas, which is consistent with the localized current conduction model in LRS.

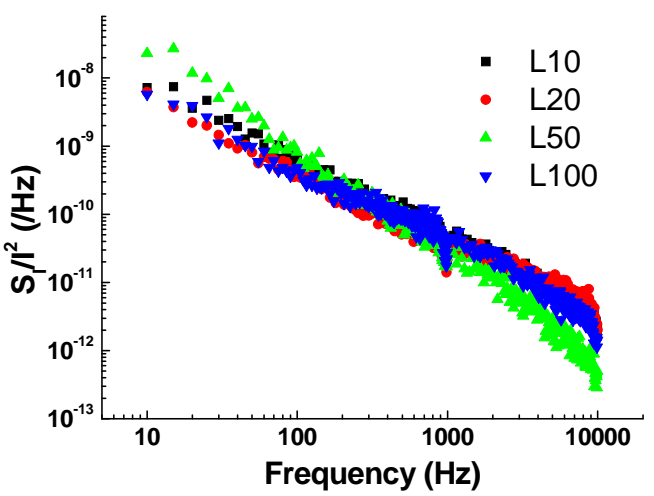

Fig. 5. Measured noise PSD of LRS state for device L10 to L100. 


\section{CONCLUSION}

Current conduction mechanism in oxide-based RRAM device has been investigated with DC measurement and LFN analysis in this work. 1/f noise behavior is observed in metal oxide based RRAM devices. Normalized noise spectrum shows no dependence on the biasing voltage, which indicates an intrinsic noise property of the current conduction. Together with DC current voltage characteristics, it is confirmed that for LRS, current conduction is localized without area dependence, whereas, for HRS, uniform leakage current scales with device area.

\section{REFERENCES}

[1] I. G. Baek, D. C. Kim, M. J. Lee, H. J. Kim, E. K. Yim, M. S. Lee, J. E. Lee, S. E. Ahn, S. Seo, J. H. Lee, J. C. Park, Y. K. Cha, S. O. Park, H. S. Kim, I. K. Yoo, U. I. Chung, J. T. Moon, and B. I. Ryu, "Multi-layer cross-point binary oxide resistive memory (OxRRAM) for post-NAND storage application," in Technical Digest - International Electron Devices Meeting, IEDM, 2005, pp. 750-753.

[2] H. Y. Lee, P. S. Chen, T. Y. Wu, Y. S. Chen, C. C. Wang, P. J. Tzeng, C. H. Lin, F. Chen, C. H. Lien, and M. J. Tsai, "Low power and high speed bipolar switching with a thin reactive ti buffer layer in robust $\mathrm{HfO} 2$ based RRAM," in Technical Digest - International Electron Devices Meeting, IEDM, 2008, pp. 297-300.

[3] D. C. Kim, S. Seo, S. E. Ahn, D. S. Suh, M. J. Lee, B. H. Park, I. K. Yoo, I. G. Baek, H. J. Kim, E. K. Yim, J. E. Lee, S. O. Park, H. S. Kim, U. I. Chung, J. T. Moon, and B. I. Ryu, "Electrical observations of filamentary conductions for the resistive memory switching in $\mathrm{NiO}$ films," Applied Physics Letters, vol. 88, 2006.
[4] Z. Fang, H. Y. Yu, W. J. Liu, Z. R. Wang, X. A. Tran, B. Gao, and J. F. Kang, "Temperature instability of resistive switching on HfOx-based RRAM devices," Electron Device Letters, IEEE, vol. 31, pp. 476-478, 2010.

[5] Y. Nishi and J. Jameson, "Challenge of nanoelectronic materials and devices toward new nonvolatile memories," in International Conference on Solid-State and Integrated Circuits Technology Proceedings, ICSICT 2008, pp. 892-896.

[6] G. Ghibaudo and T. Boutchacha, "Electrical noise and RTS fluctuations in advanced CMOS devices," Microelectronics Reliability, vol. 42, pp. 573-582, 2002.

[7] S. Machlup, "Noise in semiconductors: Spectrum of a two-parameter random signal," Journal of Applied Physics, vol. 25, pp. 341-343, 1954.

[8] M. Terai, Y. Sakotsubo, Y. Saito, S. Kotsuji, and H. Hada, "Effect of bottom electrode of ReRAM with Ta2O5/TiO2 stack on RTN and retention," in Technical Digest - International Electron Devices Meeting, IEDM, 2009, pp. 775-778.

[9] L. Jung-Kyu, J. Hu Young, C. In-Tak, L. Jeong Yong, C. Sung-Yool, K. Hyuck-In, and L. Jong-Ho, "Conduction and Low-Frequency Noise Analysis in $\mathrm{Al} / \alpha-\mathrm{TiOx} / \mathrm{Al}$ Bipolar Switching Resistance Random Access Memory Devices," Electron Device Letters, IEEE, vol. 31, pp. 603-605, 2010.

[10] S. B. Lee, S. Park, J. S. Lee, S. C. Chae, S. H. Chang, M. H. Jung, Y. Jo, B. Kahng, B. S. Kang, M. J. Lee, and T. W. Noh, "Large 1/f noise of unipolar resistance switching and its percolating nature," Applied Physics Letters, vol. 95, pp. 122112-122112-3, 2009.

[11] M. Terai, Y. Sakotsubo, Y. Saito, S. Kotsuji, and H. Hada, "Memory-State Dependence of Random Telegraph Noise of Ta2O5/TiO2 Stack ReRAM,” Electron Device Letters, IEEE, vol. 31, pp. 1302-1304, 2010. 